\title{
EFFeCT OF DiReCt SUPERVision OF A Strength CoACh on Measures of Muscular Strength AND Power in Young Rugby League Players
}

\author{
Aaron J. Coutts, ${ }^{1}$ Aron J. Murphy, ${ }^{1}$ And Ben J. Dascombe ${ }^{2}$ \\ ${ }^{1}$ School of Leisure, Sport, and Tourism, University of Technology, Sydney, Australia; ${ }^{2}$ School of Health and \\ Human Performance, Faculty of Arts, Health, and Sciences, Central Queensland University, Queensland, \\ Australia.
}

\begin{abstract}
Coutts, A.J., A.J. Murphy, and B.J. Dascombe. Effect of direct supervision of a strength coach on measures of muscular strength and power in young rugby league players. $J$. Strength Cond. Res. 18(2):316-323. 2004.-The purpose of the present study was to examine the influence of direct supervision on muscular strength, power, and running speed during 12 weeks of resistance training in young rugby league players. Two matched groups of young $(16.7 \pm 1.1$ years $[$ mean $\pm S D])$, talented rugby league players completed the same periodized resistance-training program in either a supervised (SUP) $(N=21)$ or an unsupervised (UNSUP) $(N=21)$ environment. Measures of 3 repetition maximum (3RM) bench press, 3RM squat, maximal chin-ups, vertical jump, 10- and 20-m sprints, and body mass were completed pretest (week 0), midtest (week 6), and posttest (week 12) training program. Results show that 12 weeks of periodized resistance training resulted in an increased body mass, 3RM bench press, 3RM squat, maximum number of chinups, vertical jump height, and 10- and 20-m sprint performance in both groups $(p<0.05)$. The SUP group completed significantly more training sessions, which were significantly correlated to strength increases for 3RM bench press and squat $(p<$ 0.05). Furthermore, the SUP group significantly increased 3RM squat strength (at 6 and 12 weeks) and 3RM bench press strength (12 weeks) when compared to the UNSUP group ( $p<$ 0.05 ). Finally, the percent increase in the $3 R M$ bench press, $3 R M$ squat, and chin-up max $_{\max }$ was also significantly greater in the SUP group than in the UNSUP group $(p<0.05)$. These findings show that the direct supervision of resistance training in young athletes results in greater training adherence and increased strength gains than does unsupervised training.
\end{abstract}

KEY WoRDS. strength coaching, young athletes, strength training

\section{INTRODUCTION}

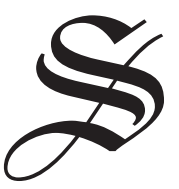

trength and conditioning coaches play an important role in athlete preparation. In particular, the strength and conditioning coach may provide support to the athlete in the form of technique analysis and modification, motivation, goal setting, and psychological reinforcement during training (5). However, while it is generally accepted that an effective strength and conditioning coach plays an important role in improving athletic performance, few studies have directly measured the influence of coaching or supervision on physiological changes or athletic performance. This is also an important consideration to make when interpreting research. In particular, it may be difficult to determine whether the physiological and perfor- mance changes reported in previous studies are due to the type and level of supervision of training.

To date, there has been 1 published study that has examined the influence of one-on-one personal trainer supervision during a 12 -week periodized resistance-training program (19). These investigators compared the changes in physical and strength characteristics between matched groups of supervised (SUP) and unsupervised (UNSUP) highly motivated male strength trainers. Results from this previous study showed that the SUP group recorded significantly increased squat and bench press maximal strength when compared to the matched UNSUP group. There were no significant changes between the groups in vertical jump and body mass measures. Both the SUP and UNSUP groups completed the same amount of training in terms of sets and repetitions; however, the SUP group trained with heavier training weights. The investigators suggested that the increases in strength were attributable to the increased training intensities completed by the SUP group. It was suggested that the increased motivation and competitiveness provided by the personal trainers facilitated increased training intensity and strength development. The findings of this recent study are important for both strength coaches working in an applied setting and researchers working in a clinical setting, as they show that the level of supervision during resistance training can influence strength performance and physiological measures.

It is recommended that the level of supervision for athletes be modified according to experience and lifting ability of the athlete $(1,2,8,14)$. For example, it is the position of the National Strength and Conditioning Association (NSCA) that the athlete-to-supervisor ratio be 1:10 for junior high school athletes, 1:15 for high school athletes, and 1:20 for experienced college athletes (8). However, although the NSCA has developed recommendations for the supervision of resistance training, at present, there is a paucity of data on the influence of supervision on physiological and performance changes with resistance training in both the research and field settings. Furthermore, although the NSCA recommends that young athletes complete strength training while under the supervision of a qualified strength and conditioning coach $(8,10)$, the influence of supervision on performance in young athletes has not been examined. Therefore, the purpose of this study was to examine the influence of di- 
TABLE 1. Subject characteristics (mean $\pm S D)$.*

\begin{tabular}{lcccc}
\hline \multicolumn{1}{c}{ Group } & $\begin{array}{c}\text { Age } \\
\text { (years) }\end{array}$ & $\begin{array}{c}\text { Height } \\
(\mathrm{cm})\end{array}$ & $\begin{array}{c}\text { Body mass } \\
(\mathrm{kg})\end{array}$ & $\begin{array}{c}\text { Training } \\
\text { experience } \\
(\mathrm{months})\end{array}$ \\
\hline UNSUP $(n=21)$ & $16.6 \pm 1.2$ & $168.0 \pm 6.4$ & $74.7 \pm 8.6$ & $3.1 \pm 4.5$ \\
SUP $(n=21)$ & $16.8 \pm 1.0$ & $170.0 \pm 5.4$ & $77.9 \pm 8.7$ & $3.4 \pm 5.6$ \\
\hline
\end{tabular}

* UNSUP $=$ unsupervised; SUP $=$ supervised.

rect supervision on muscular strength, power, and running speed during a 12 -week resistance-training program with young rugby league players.

\section{Methods}

\section{Experimental Approach to the Problem}

There is limited information on the influence of supervision during strength training on physiological and performance changes both in the applied sports training setting and during scientific research studies. The hypothesis of the current study is that supervised resistance training leads to increased muscular strength and power in young athletes. The present research examined the changes in various physiological and performance measures of strength and power in 2 matched groups of young rugby league players. Each group completed the same 12week resistance-training program (a) under the supervision of a trained strength and conditioning coach, or (b) without direct supervision. Statistical analyses of pretraining and posttraining measures allowed us to evaluate the influence of supervision on changes in physiological and performance measures during the same 12-week resistance-training program.

\section{Subjects}

Forty-two healthy young $(16.7 \pm 1.1$ years $[$ mean $\pm S D])$ male rugby league players participated in the study $(21$ subjects in both years of the study). All subjects were talented players and were participating in a regional rugby league development program. Table 1 shows the subjects' physical characteristics. Prior to the commencement of the study, all players received a clear explanation of the study that informed them of all risks and benefits associated with participation, and informed consent was obtained both from the players and their legal guardians. Central Queensland University Human Ethics Review Panel approval was given for all experimental procedures.

\section{Experimental Procedures}

Study Design. Two independent groups of talented rugby league players that were carefully matched for age, training history, and physiological characteristics completed the same resistance-training program in either a SUP or an UNSUP training environment (Table 1). The SUP group completed training 12 months after the UNSUP program. Physiological testing was completed pretest (week 0), midtest (week 6), and posttest (week 12) training program using standardized equipment and procedures. The performance and physiological tests included a 3RM bench press, a 3RM squat, a maximal number of chin-ups (chin-up max $_{\text {mas }}$, a vertical jump, 10- and 20-m sprints, and body mass measures. These performance and physiological tests were selected because they are com-
TABLE 2. Resistance training exercises completed during the 12-week training program.

\begin{tabular}{ll}
\hline \multicolumn{1}{c}{$\begin{array}{c}\text { Phase 1 } \\
\text { (weeks 0-6) }\end{array}$} & \multicolumn{1}{c}{$\begin{array}{c}\text { Phase 2 } \\
\text { (weeks 7-12) }\end{array}$} \\
\hline $\begin{array}{l}\text { Back squat* } \\
\text { Wide grip pulldown behind* }\end{array}$ & $\begin{array}{l}\text { Box jumps } \\
\text { Back squat* } \\
\text { Bench press* }\end{array}$ \\
Front military press* & Bench pull (to waist)* \\
Shoulder dips (weighted) & Push press \\
Abdominal crunches & Chin-ups (weighted) \\
Back extension & Abdominal crunches \\
& Hamiglut raise \\
\hline
\end{tabular}

\footnotetext{
* Training load prescribed at the start of training phase using the methods of Baker (3).
}

monly used to monitor changes in strength and power in young rugby league players $(6,13)$. Reliability data were collected for each of the dependent variables prior to the training program.

Familiarization. In the week prior to the commencement of the training period, all subjects were screened for contraindications to exercise and gave informed consent. At this time, all subjects were assessed for physical characteristics and muscle strength and power, were coached on proper lifting techniques, and were instructed on the important parameters of the training program. Factors such as exercise technique, equipment use, exercise order, lifting tempo, rest periods between sets, and program progression were carefully explained. Later educational sessions were also presented to each group of subjects after 6 weeks of training. These education sessions were used to reinforce basic strength-training principles.

Resistance-Training Program. Each group was prescribed the same 12-week periodized resistance-training program during the study. Training was completed 3 days per week on Monday, Wednesday, and Friday for both groups. The resistance-training exercises prescribed during the study are shown in Table 2. The weight on the bar for core exercises was calculated according to the methods suggested by Baker (3) in both training periods. The players were encouraged to adjust these weights if they were too heavy or too light. When training loads were altered, players were instructed to inform the chief investigator. A detailed description of the resistancetraining periodization is shown in Table 3.

Training Supervision. During the UNSUP-training period, all resistance-training sessions were supervised by a team manager who was not trained in strength and conditioning coaching. The role of the manager during this period was to monitor training attendance and program administration. All training sessions were completed in the same 3 commercial fitness centers where gym instructors were available for technical feedback and in- 
TABLE 3. Description of prescribed resistance training completed by the SUP and UNSUP groups during the 12 -week training period.*

\begin{tabular}{lcccccccccccc}
\hline \multicolumn{1}{c}{ Week } & 1 & 2 & 3 & 4 & 5 & 6 & 7 & 8 & 9 & 10 & 11 & 12 \\
\hline Repetitions & 16 & 14 & 13 & 12 & 11 & 10 & 9 & 8 & 7 & 6 & 5 & 4 \\
Sets & 17 & 17 & 20 & 22 & 22 & 20 & 18 & 18 & 22 & 22 & 24 & 24 \\
Intensity (\%1RM) & 55.0 & 60.0 & 62.5 & 68.5 & 71.0 & 73.5 & 76.0 & 78.5 & 81.0 & 83.5 & 86.0 & 88.5 \\
Rest period (min) & 1 & 1 & 1 & 1 & 1 & 1 & 2.5 & 2.5 & 2.5 & 2.5 & 2.5 & 2.5 \\
Sessions per week & 3 & 3 & 3 & 3 & 3 & 3 & 3 & 3 & 3 & 3 & 3 & 3 \\
\hline
\end{tabular}

$* \mathrm{RM}=$ repetition maximum; SUP $=$ supervised; UNSUP $=$ unsupervised.

struction when requested. The mean attendance during this training period was $84.7 \pm 15.1 \%$ of all sessions.

Twelve months following the commencement of the first training period, the SUP group completed the same periodized resistance-training program under the direct supervision of a trained strength and conditioning coach (Australian Strength and Conditioning Association, Level One). The coach-to-athlete ratio was 1:7. The attendance during this training period was $94.5 \pm 5.1 \%$ of all sessions. No injuries occurred in any of the subjects during the completion of this study.

\section{Physiological Assessment}

Anthropometry. Body mass was measured postmicturition using electronic scales (Mercury, Australia) accurate to $50 \mathrm{~g}$. The same scales were used on each testing occasion. Each subject's height was measured by a trained anthropometrist using a standard stadiometer (Blaydon Stadiometer, Lugarna, Australia). The stretch stature method was used with the subject's feet together and the heels, buttocks, and upper part of the back touching the scale with the subject's head when placed in the Frankfort Plane (25).

Vertical Jump. Vertical jump height was assessed using a Vertec jumping device (Sports Imports, Columbus, $\mathrm{OH})$. Each subject stood side-on to the Vertec with his heels placed on the ground. Prior to each test jump, the subjects were asked to reach upward as high as possible, fully elevating the shoulder to displace the zero-reference vane. The take-off was from $2 \mathrm{ft}$ with no preliminary steps or shuffling. An arm swing and a countermovement were used, with the subject jumping as high as possible, displacing the vane. The height of the jump was calculated as the distance from the highest vane reached and the zero-reference vane. Each subject performed 3 trials, and the best of these trials was recorded. The reliability of vertical jump measures for this laboratory was high (intraclass correlation coefficient [ICC] $r=0.97$; percent technical error of measurement [TEM\%]: 2.12).

Muscle Strength Testing. Muscle strength testing included a standardized warm-up that was followed by a 3RM parallel squat, a 3RM bench press, and a chin-up maximum (chin-up max $_{\text {ax }}$ ). The $3 R M$ tests were chosen in preference to the 1RM as a measure of strength because of the relative inexperience of the subjects and also because previous normative data for young rugby league players exist for these measures (12). The 3RM parallel squat and bench press testing procedures included 2-3 warm-up sets of 5-8 repetitions with light-to-moderate resistance. A successful parallel squat required the thigh to descend to a parallel position in which the trochanter head of the femur was in the same horizontal plane as the superior border of the patella (23). A successful bench press required the bar to be slowly lowered to the chest of the subject and returned to full extension of both elbow joints. The subjects were required to keep their hips and feet on the bench and floor, respectively, at all times during each lift attempt. The reliability of the parallel squat (ICC: $r=0.96$; TEM\%: 2.32) and bench press (ICC: $r=$ 0.98; TEM\%: 1.46) measures for this group was high.

The chin-up max $_{\max }$ test was completed at least 10 minutes after the bench press test. During this test, the subjects were instructed to attempt as many full, unassisted chinups as possible until volitional fatigue. Each chin-up was completed on a horizontal bar raised $2.5 \mathrm{~m}$ above the floor. A successful chin-up required the subject to start with his chin above the horizontal bar and to lower his body until full extension in both elbows and then lift his body weight until his chin returned to the starting position. If assistance was offered by a spotter during any lifting attempt, then the subject was instructed to stop. Subjects were allowed 3 chin-up max $_{\text {mials, }}$, and the highest number of repetitions was recorded. The reliability of the chin-up max $_{\max }$ test (ICC: $r=0.99$; TEM\%: 2.57) for this group was high.

Speed. Running speed was assessed by 10- and 20-m sprint times using electronic timing gates (Swift, Goonellabah, Australia). Timing gates were positioned in a straight direction at 10 and $20 \mathrm{~m}$ from a marked starting point. On an audible command, the players sprinted as quickly as possible along the $20-\mathrm{m}$ course. Time to cover the $10-$ and $20-\mathrm{m}$ distances was measured to the nearest 0.01 second, with the faster of 2 trials being recorded. The reliability of the 10-m (ICC: $r=0.91$ and TEM\% 2.02) and the 20-m (ICC: $r=0.91$ and TEM\% 1.86) sprint tests was high.

\section{Statistical Analyses}

The means and $S D$ were calculated for each of the dependent variables. The data were analyzed using a two-way analysis of variance (ANOVA) with a repeated-measures design, and a Tukey honestly significant difference post hoc comparison was applied to determine significant differences over time. A one-way ANOVA was used to determine significant differences between the groups in percent change. Pearson correlation coefficients were determined for selected variables. The SPSS statistical software package, version 11 (SPSS Inc., Chicago, IL), was used for statistical calculations. The level of significance was set at 0.05 .

\section{Results}

\section{Training}

The UNSUP group completed significantly fewer training sessions $(55.9 \pm 10.0$ sessions) than the SUP group (62.4 
TABLE 4. Body mass $(\mathrm{kg})$ changes during 12 weeks of resistance changes (mean $\pm S D)$.

\begin{tabular}{lccc}
\hline \multicolumn{1}{c}{ Group } & $\begin{array}{c}\text { Pre } \\
\text { (week 0) }\end{array}$ & $\begin{array}{c}\text { Mid } \\
(\text { week 6) }\end{array}$ & $\begin{array}{c}\text { Post } \\
\text { (week 12) }\end{array}$ \\
\hline UNSUP $(n=21)$ & $74.7 \pm 8.6$ & $75.6 \pm 8.9 \dagger$ & $76.8 \pm 9.6^{* \dagger}$ \\
SUP $(n=21)$ & $77.9 \pm 8.7$ & $79.7 \pm 10.0 \dagger$ & $80.8 \pm 10.4^{* \dagger}$ \\
\hline
\end{tabular}

* Significantly different from previous measure $(p<0.05)$.

$\dagger$ Significantly different from pretest measure $(p<0.05)$.

$\ddagger$ UNSUP $=$ unsupervised; SUP $=$ supervised

\pm 3.3 sessions $)(p<0.05)$. A significant correlation was observed between training attendance and percent changes in the 3RM bench press $(r=0.35, p<0.05)$ and the $3 \mathrm{RM}$ squat measures $(r=0.35, p<0.05)$.

\section{Body Mass}

Table 4 shows the body mass changes in both groups during the 12 weeks of resistance training. During the 12 weeks of resistance training, the body mass was significantly increased by $2.8 \pm 1.6 \%$ and $3.6 \pm 3.0 \%$ in the UNSUP and SUP groups, respectively. However, there was no significant difference in the amount of change in body mass between the UNSUP and SUP groups.

\section{Muscular Strength, Power, and Running Speed}

Table 5 shows changes in muscular strength, power, and running speed during the 12 weeks of SUP or UNSUP periodized resistance training in young rugby league players. The $3 \mathrm{RM}$ bench press was increased significantly at midtesting and posttesting occasions when compared to the pretest values in both the UNSUP and SUP groups $(p<0.05)$. Additionally, the absolute 3RM bench press was significantly increased in the SUP group when compared to the UNSUP group at the posttesting occasion ( $p$
$<0.05)$. Mean percent change increase in the bench press for the UNSUP and SUP groups during the 12 weeks of resistance training was $15.3 \pm 6.9 \%$ and $29.8 \pm 8.9 \%$, respectively. When compared to the UNSUP group, the SUP group experienced significantly greater percent gains in bench press strength following 12 weeks of resistance training $(p<0.05)$.

The 3RM squat was increased significantly at midtesting and posttesting occasions when compared to the pretraining measures in both the UNSUP and SUP groups $(p<0.05)$. Additionally, the absolute 3RM squat was significantly increased in the SUP group when compared to the UNSUP group at the midtesting and posttesting occasions $(p<0.05)$. Mean percent change increases in the squat for the UNSUP and SUP groups during the 12 weeks of resistance training was $25.5 \pm$ $15.8 \%$ and $40.1 \pm 26.9 \%$, respectively. The SUP group experienced significantly greater percent gains in squat strength during the 12-week period than did the UNSUP group $(p<0.05)$.

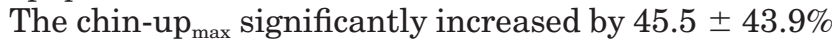
and $97.0 \pm 105.9 \%$ in the UNSUP and SUP groups, respectively, after the 12 weeks of periodized resistance training. No significant differences were observed between groups in the chin-up max $_{\max }$ at the pretraining, midtraining, or posttraining periods. However, the percent change for the chin-up $p_{\max }$ was significantly greater in the SUP group $(p<0.05)$ (Figure 1).

The vertical jump increased significantly by $6.7 \pm$ $3.9 \%$ and $9.7 \pm 7.9 \%$ in the UNSUP and SUP groups, respectively, after the 12 weeks of resistance training. No significant differences between groups were observed in absolute increase or percent change in vertical jump measures during the 12 -week resistance training.

The 10-m sprint times decreased significantly by 1.1 $\pm 0.9 \%$ and $1.2 \pm 0.7 \%$ in the UNSUP and SUP groups,

TABLE 5. Performance changes during the 12-week resistance-training program.

\begin{tabular}{|c|c|c|c|}
\hline Group & $\begin{array}{c}\text { Pre } \\
\text { (week 0) }\end{array}$ & $\begin{array}{c}\text { Mid } \\
\text { (week 6) }\end{array}$ & $\begin{array}{c}\text { Post } \\
\text { (week 12) }\end{array}$ \\
\hline \multicolumn{4}{|l|}{ 3RM bench press $(\mathrm{kg})$} \\
\hline UNSUP & $68.9 \pm 13.0$ & $74.5 \pm 13.1 \dagger$ & $79.0 \pm 13.2^{* \dagger}$ \\
\hline SUP & $69.9 \pm 11.1$ & $81.1 \pm 11.8 \dagger$ & $90.2 \pm 12.6^{*}+\ddagger$ \\
\hline \multicolumn{4}{|l|}{ 3RM squat (kg) } \\
\hline UNSUP & $79.3 \pm 19.9$ & $89.9 \pm 18.2 \dagger$ & $97.5 \pm 17.6^{* \dagger}$ \\
\hline SUP & $87.6 \pm 19.3$ & $112.4 \pm 21.1 \dagger+$ & $120.2 \pm 22.2^{*}+\dagger$ \\
\hline \multicolumn{4}{|l|}{ Chin-up max $_{\text {mo.) }}$} \\
\hline UNSUP & $8.9 \pm 4.2$ & $10.8 \pm 4.4 \dagger$ & $12.0 \pm 4.4^{* \dagger}$ \\
\hline SUP & $6.9 \pm 4.2$ & $8.7 \pm 3.9 \dagger$ & $11.2 \pm 4.7^{* \dagger}$ \\
\hline \multicolumn{4}{|l|}{ Vertical jump (cm) } \\
\hline UNSUP & $50.3 \pm 6.9$ & $51.7 \pm 6.6 \dagger$ & $53.5 \pm 6.5^{* \dagger}$ \\
\hline SUP & $51.1 \pm 6.6$ & $53.5 \pm 5.8 \dagger$ & $55.7 \pm 5.5^{* \dagger}$ \\
\hline \multicolumn{4}{|l|}{ 10-m sprint (ss.ms) } \\
\hline UNSUP & $02.14 \pm 00.09$ & $02.13 \pm 00.08 \dagger$ & $02.12 \pm 00.08^{* \dagger}$ \\
\hline SUP & $02.16 \pm 00.08$ & $02.14 \pm 00.09 \dagger$ & $02.14 \pm 00.08^{*}$ \\
\hline \multicolumn{4}{|l|}{ 20-m sprint (ss.ms) } \\
\hline UNSUP & $03.47 \pm 00.11$ & $03.44 \pm 00.10 \dagger$ & $03.43 \pm 00.09 * \dagger$ \\
\hline SUP & $03.46 \pm 00.08$ & $03.45 \pm 00.08 \dagger$ & $03.43 \pm 00.08 * \dagger$ \\
\hline
\end{tabular}

* Significantly different from previous measure $(p<0.05)$.

$\dagger$ Significantly different from pretest $(p<0.05)$.

$\ddagger$ Significantly different from UNSUP $(p<0.05)$.

$\S \mathrm{RM}=$ repetition maximum; UNSUP $=$ unsupervised $(n=21) ; \mathrm{SUP}=\operatorname{supervised}(n=21 ;$ mean $\pm S D)$. 


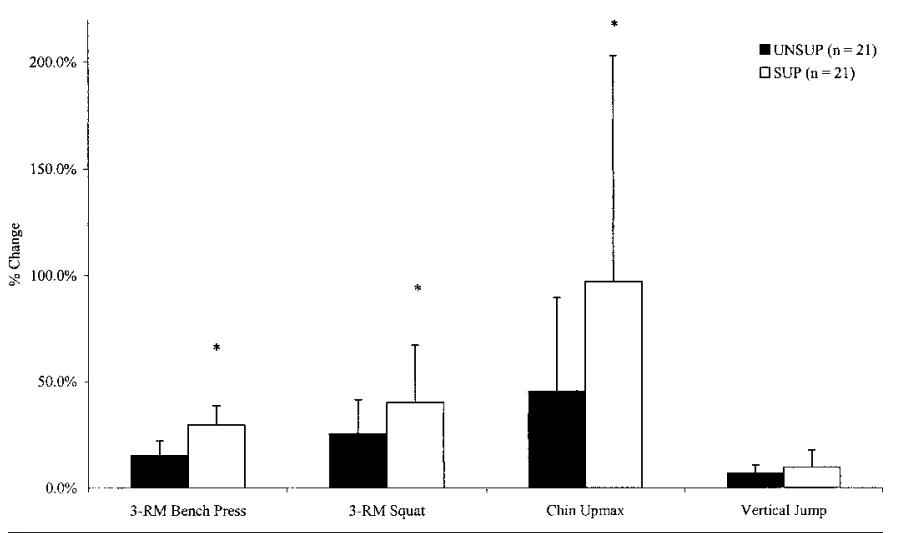

FIGURE 1. Percentage of change in the muscular strength and power measures during 12 weeks of supervised (SUP) or unsupervised (UNSUP) resistance training. *Significantly different from the UNSUP group $(p<0.05)$.

respectively, during the 12 weeks of resistance training. Similarly, the $20-\mathrm{m}$ sprint times decreased significantly by $1.2 \pm 1.2 \%$ and $0.8 \pm 0.6 \%$ in the UNSUP and SUP groups, respectively, during the study. No significant differences in absolute decrease or percent change were measured in the 10- and 20-m sprint times between groups.

\section{Discussion}

This study investigated the influence of direct supervision on measures of muscular strength, power, and running speed during 12 weeks of resistance training in young rugby league players. The main finding of the present study was that direct supervision significantly increased the absolute 3RM bench press and 3RM squat strength after 12 weeks of resistance training in comparison to a matched group that completed the same resistance-training program, unsupervised. Furthermore, although both training groups increased absolute muscular strength, power, running speed, and body mass during the 12 weeks of resistance training, the SUP group demonstrated a significantly greater percentage of improvement than the UNSUP group in the bench press, squat, and chin- up $_{\max }$ tests. The SUP group completed significantly more training sessions than the UNSUP group. To date, there has been only 1 comparable study, to our knowledge, that has investigated the influence of supervision on measures of muscular strength and power (19). However, this earlier study examined the responses to supervised training in 10 men (aged $24.6 \pm 1.0$ years) who were very experienced in resistance training. In contrast to this previous research, the present study investigated young athletes with relatively little resistance-training experience.

The present study demonstrated that the absolute $3 \mathrm{RM}$ bench press and squat strength was significantly increased after 6 and 12 weeks of resistance training in both UNSUP and SUP environments in young rugby league players (Table 5). The significant differences in absolute strength between groups in the squat were identified after 6 weeks of training, whereas the differences between groups for the bench press were found after 12 weeks of training. The increases in the bench press and squat strength in the SUP group were most likely due to the increased total training loads completed during the training period. In particular, it appears that increased training frequency and possibly an increased daily training intensity contributed to the increased strength adaptations in the SUP group. This suggestion is supported by the present finding of a significant relationship between training attendance and increases in strength for both the bench press $(r=0.35, p<0.05)$ and the squat $(r=0.35, p<0.05)$.

The resistance training prescribed for the subjects in the present study was the same for both experimental groups, with the exception of training attendance rates and possibly mean training load. Both of these factors may explain the increased strength gains with supervision. Although daily training intensity was not accurately recorded in the present study, previous research suggests that training intensity is increased with direct supervision (19). For example, Mazzetti et al. (19) reported that direct supervision by personal trainers resulted in accelerated progression in training loads during 12 weeks of linear periodized resistance training in 10 men experienced in resistance training. These investigators suggested that the increased tolerance of heavy training loads leads to the stimulation of higher threshold motor units (27). Therefore, we suggest that both the observed increase in training frequency and the previously reported increase in training intensity (19) are the factors that led to the improvement in muscle strength with direct supervision in this study.

A possible explanation for the accelerated increase in the squat strength gains when compared to the bench press strength gains is that the young rugby league players had learned the technical skill of squatting. Previous authors have reported that resistance training in young athletes increases motor performance and lifting technique (10). The influence of direct supervision and increased coaching by the supervisors most probably improved squatting techniques early in the program for the SUP group and is therefore the most likely explanation for the early differences in measures after 6 weeks of training.

The magnitude of the relative strength increases in the present study appears to be larger than those observed by previous researchers investigating the effect of direct supervision on muscular strength and power adaptations (19). Mazzetti et al. (19) reported relative strength increases in both the 1RM squat and bench press of SUP strength trainers to be $33.0 \pm 4.2 \%$ and 22.0 $\pm 2.2 \%$, respectively, which are less than those measured in the present study. We suggest that the increased strength gains in the present study are primarily due to accelerated neural adaptations and the learning of lifting techniques. This may explain the difference in the magnitude of relative strength changes: the experienced lifters in the previous study would have already learned the lifting skills and gained most of the benefit of the neural changes associated with commencing strength training. Additionally, the young athletes of the present study were at the approximate stage of maturation reported to be associated with accelerated strength improvements, which may have contributed to the increased strength gains in the present study (18).

Previous studies of young athletes have reported strength increases of up to $76 \%$ (10). However, adoles- 
cents are more likely to increase strength by 20 to $50 \%$ with carefully planned, short-term resistance training (520 weeks) like that in the present study $(24,28,30,33$, 34). The strength increases measured in the present study are comparable to these previous findings. In addition to these strength increases in both conditions, there was a significantly increased percent change in the 3RM squat, bench press, and chin-up max $_{\max }$ in the SUP group when compared to the UNSUP group. These findings further support the efficacy of the direct supervision of resistance training in young athletes. We suggest that the gains observed in the present study are due to both neural and hypertrophic adaptations, which are increased with direct supervision. Neural adaptations such as increased recruitment of muscle fibers, increased speed of recruitment, improved synchronization of muscle recruitment, and decreased muscle inhibition have been suggested to be the most significant contributors to improvement in strength performance in short-term training studies (8-12 weeks) and in untrained athletes (7, 22, 29). Considering the low level of resistance-training experience in the present cohort (Table 1) and the findings from previous studies $(7,22,29)$, it seems reasonable to suggest that these neurological adaptations are contributors to the increases in strength observed during the present study.

In addition to neural adaptations, it is suggested that muscle hypertrophy contributed to the increases in strength. This suggestion is somewhat supported by the increased body mass during the 12-week study in both groups. Previous studies have shown that high-intensity resistance training increases the hypertrophic responses in studies of relatively short duration $(11,16,31,32)$. For example, Fry et al. (11) reported increased muscle hypertrophy in 32 college-aged students during 8 weeks of twice-weekly resistance-training sessions (1 session of 6 $8 \mathrm{RM}$ and 1 session of $8-10 \mathrm{RM})$. In this previous study in which the training was similar to that in the current study, the muscle fiber area of type II fibers was hypertrophied twice as much as type I fibers. These previous findings indicate that some muscle hypertrophy had occurred in the type II fibers of the athletes in the present study.

In accordance with strength measures, muscular power measures of vertical jump and 10- and 20-m sprint times were enhanced significantly with 12 weeks of periodized resistance training in both the UNSUP and SUP groups. However, in contrast to the strength measures, there were no between-group differences in these measures (Table 5). The lack of difference between the UNSUP and SUP groups was not unexpected, as the training was not specific to sprinting, and increased sprint times were not the primary goals of the periodized resistance training. Furthermore, the resistance training was designed so that the athletes completed heavy lifts at low velocity, which is at the opposite end of the force-velocity curve to sprinting.

The enhancement in the 10- and 20-m sprint performances is similar to that observed in previous research examining the influence of resistance training on sprint performances in young athletes (21) and mature athletes $(9,23)$. For example, an 8-week resistance-training program in 30 well-trained recreational athletes demonstrated a $2.3 \%$ increase in sprint performance (23). Furthermore, the sprint times at the conclusion of the 12 -week program in this study are similar to those reported in semiprofessional rugby league players (12) and are faster than those reported in young club-level rugby league players (13). This suggests that resistance training is an important aspect in the athletic preparation of young rugby league players. Similar to the other measures of muscular power in the present study (vertical jump) and the measures of muscular power in previous studies (19), sprint times improved with 12 weeks of heavy resistance training, with no differences with direct supervision.

The observed changes in measures of muscular power are in accordance with previous research (19), which also reported increased power measured during a jump squat with 12 weeks of periodized resistance training but no effect of supervision. These previous investigators suggest that the lack of change between groups in power output was due to the lack of specificity in the resistance-training program. Accordingly, it is suggested that future studies examine the influence of supervised sprint, power, and plyometric training on measures of muscular performance.

Body mass increased significantly after 12 weeks of training in both groups. These results agree with previous research that reported increased body mass, fat mass, and fat-free mass with 12 weeks of supervised resistance training (19). However, in contrast to this previous study, similar changes were observed in both the SUP and UNSUP groups. Considering the age of the subjects in the present study, it seems reasonable to suggest that some of the increase in the body mass measures in both groups of the present study was due to normal changes associated with puberty $(15,17)$. Maturation effects may also partially explain why the relative changes in strength and power in the present study are greater than those previously reported in experienced strength trainers (19). However, because body composition was not directly assessed, an accurate comment on the mechanism of body mass change is difficult.

Success in sports such as rugby league may be achieved by increasing inertia and momentum, which may be due to the body mass gain associated with resistance training $(12,20,26)$. The increase in body mass in the present study is fundamentally important to young rugby league players, as increased body mass has been shown to be associated with rugby league performance in some studies $(6,12)$. Furthermore, earlier research suggests that players with increased body mass are more suitable to playing rugby league as a forward $(12,20,26)$. Therefore, it appears that the direct supervision of resistance training in young rugby league players may contribute to increased performance, particularly in young forwards.

In agreement with previous research (19), the present results show that the direct supervision of a 12 -week periodized resistance training by a trained strength and conditioning coach will increase the rate of strength adaptation in young athletes. It appears that increases in strength adaptation are related to increased training attendance and, possibly, increased training intensity. Future studies should examine the effect of the optimal amount and type of supervision on the development of muscular size, strength, power, and endurance as well as sports-specific performance. Of particular interest to rugby league strength and conditioning coaches is the effect of supervision on sport-specific power. This is of particu- 
lar relevance for these athletes and coaches, as recent studies investigating differences between professional and semiprofessional rugby league players demonstrated that increased power differentiated the elite from the subelite rugby league players $(4,6)$.

\section{Practical Applications}

Previous studies suggest that a lack of supervision results in inadequate workout quality (14). It has recently been reported that mean daily training loads and muscular strength and power were increased with direct supervision (19). In support of these findings, the present investigation demonstrates that training adherence and muscular strength are also improved with the direct supervision of a qualified strength and conditioning coach. The present findings provide strong support for the direct supervision of resistance training in young athletes. In the practical setting, the direct supervision of resistance training by appropriately qualified strength and conditioning coaches will significantly augment the absolute strength and percent increases of supervised athletes when compared to unsupervised athletes. Improvements in strength are of considerable benefit to young athletes in many sports, because stronger, faster, and bigger athletes have been suggested to perform at an increased level $(6,12,13)$. Additionally, the present results have important applications for scientific studies examining the benefits of resistance straining. These results indicate that future studies should accurately control and report on the type and level of supervision of resistance training, because supervision can influence physiological and performance results independent of the training program. Finally, the direct supervision of training sessions by trained strength and conditioning coaches may offer other advantages to athletes, such as improved technical feedback, increased safety, increased motivation, and increased competitiveness at training.

\section{REFERENCES}

1. Armitage-Johnson, S. Safety tips: Providing a safe training environment, part II. Strength Cond. 16:34. 1994.

2. BAEChle, T.R., AND R.W. EARLE. Essentials of Strength Training and Conditioning (2nd ed.). Champaign, IL: Human Kinetics, 2000 .

3. BAKER, D. Designing, implementing and coaching strength training programs for beginner and intermediate level athletes-Part 2: Implementing the program. Strength Cond. Coach. 5:2-8. 1997.

4. BAKER, D. Comparison of upper-body strength and power between professional and college-aged rugby league players. $J$. Strength Cond. Res. 15:30-35. 2001.

5. BAKER, D. Science and practice of coaching a strength training program for novice and intermediate-level athletes. Strength Cond. J. 23:61-68. 2001.

6. BAKER, D. Differences in strength and power among juniorhigh, senior-high, college-aged, and elite professional rugby league players. J. Strength Cond. Res. 16:581-585. 2002.

7. BEHM, D.G. Neuromuscular implications and applications of resistance training. J. Strength Cond. Res. 9:264-274. 1995.

8. Brass, M., J. Eickhoff-ShemeK, B. Epley, D. Herbert, J. Owens, D.R. Pearson, S. Plisk, And D. Wathen. Strength and Conditioning Professional Standards Guidelines. Colorado Springs, CO: NSCA, 2001. p. 48.

9. Dintiman, G.B. Effects of various training programs on running speed. Res. Q. 35:456-463. 1964.

10. Faigenbaum, A.D., W.J. Kraemer, B. Cahill, T.J. Chandler, J. Dziados, L.D. Elfrink, E. Forman, M. Gaudiose, L. Mich-
ELI, M. NikTKA, AND S. RoberTs. Youth resistance training: Position statement paper and literature review. Strength Cond. 62:62-75. 1996.

11. Fry, A.C., C.A. Allemeier, ANd R.S. Staron. Correlation between percentage fiber type area and myosin heavy chain content in human skeletal muscle. Eur. J. Appl. Physiol. 68:246251. 1994.

12. GABBETT, T.J. Influence of physiological characteristics on selection in a semi-professional first grade rugby league team: A case study. J. Sports Sci. 20:399-405. 2002.

13. GABBETT, T.J. Physiological characteristics of junior and senior rugby league players. Br. J. Sports Med. 36:332-339. 2002.

14. Hillmann, A., AND D.R. Pearson. Supervision: The key to strength training success. Strength Cond. 17:67-71. 1995.

15. ISRAEL, S. Age-related changes in strength and special groups. In: Strength and Power in Sport. P.V. Komi, ed. Oxford, UK: Blackwell Science Ltd., 1992. pp. 319-328.

16. JoNES, D.A., AND O.M. RUTHERFORD. Human muscle strength training: The effects of three different regimes and the nature of the resultant changes. J. Physiol. 391:1-11. 1987.

17. Kraemer, W.J., A.C. Fry, P.N. Frykman, B. Conroy, And J. Hoffman. Resistance training and youth. Pediatr. Exerc. Sci. 1:336-350. 1989.

18. Malina, R., AND C. Bouchard. Growth, Maturation and Physical Activity. Champaign, IL: Human Kinetics, 1991. pp. 87150.

19. Mazzetti, S.A., W.J. Kraemer, J.S. Volek, N.D. Duncan, R.A. Ratamess, A.L. Gomez, R.U. NEwTon, K. HÄKKINEN, AND S.J. FLECK. The influence of direct supervision of resistance training on strength performance. Med. Sci. Sports Exerc. 32:11751184. 2000.

20. Meir, R., R.U. Newton, E. Curtis, M. Fardell, and B. ButLER. Physical fitness qualities of professional rugby league football players: Determination of positional differences. $J$. Strength Cond. Res. 15:450-458. 2001.

21. Mero, A. Neuromuscular performance and skeletal muscle fibre characteristics in pubertal power athletes during training. Med. Sci. Sports Exerc. 25:s47. 1993.

22. Moritani, T., AND H.A. DEVRIEs. Neural factors versus hypertrophy in the time course of strength gain. Am. J. Phys. Med. 58:115-130. 1979.

23. Murphy, A.J., AND G.J. Wilson. The ability of tests of muscular function to reflect training-induced changes in performance. J. Sports Sci. 15:191-200. 1997.

24. Nielson, B., K. Nielson, M. Behrendt-Hansen, and E. AsMUSSEN. Training of "functional muscular strength" in girls 719 years old. In: Children and Exercise IX. K. Berg and B. Eriksson, eds. Baltimore, MD: University Park Press, 1980. pp. 69-77.

25. Norton, K., M. Marfell-Jones, N. Whittingham, D. KerR, L. Carter, K. Saddington, And C. Gore. Anthropometric assessment protocols. In: Physiological Testing the Elite Athlete. C.J. Gore, ed. Champaign, IL: Human Kinetics, 2000. pp. 66-85.

26. O'Connor, D. Physiological characteristics of professional rugby league players. Strength Cond. Coach. 4:21-26. 1996.

27. Ploutz, L.L., P.A. Tesch, R.L. Biro, and G.A. Dudley. Effect of resistance training on muscle use during exercise. J. Appl. Physiol. 76:1675-1681. 1994.

28. Ramsay, J., K. Blimkie, S. Smith, S. Garner, J.D. MaCDougall, AND D.G. SALE. Strength training effects in prepubescent boys. Med. Sci. Sports Exerc. 22:605-614. 1990.

29. SALE, D.G. Neural adaptation to resistance training. Med. Sci. Sports Exerc. 20:s135-145. 1988.

30. SALE, D.G. Strength training in children. In: Perspectives in Exercise Science and Sports Medicine. G.V. Gisolfi and D.R. Lamb, eds. Indianapolis, IN: Benchmark, 1989. pp. 165-216.

31. SAlE, D.G., J.E. MARTin, AND D.E. Moroz. Hypertrophy without increased isometric strength after weight training. Eur. J. Appl. Physiol. 64:51-55. 1992.

32. Staron, R.S., D.L. Karapondo, W.J. Kraemer, A.C. Fry, S.E. Gordon, J.E. FAlKel, F.C. HAGERMan, AND R.S. HikidA. Skel- 
etal muscle adaptations during early phase of heavy-resistance training in men and women. J. Appl. Physiol. 76:1247-1255. 1994.

33. VRIJENS, F. Muscle strength development in the pre- and postpubescent age. Med. Sport. 11:152-158. 1978.

34. WROBLE, R.R., AND D.P. MoXLEY. The effect of winter sports participation on high school football players: Strength, power, agility, and body composition. J. Strength Cond. Res. 15:132135. 2001.

Address correspondence to Dr. Aaron Coutts, aaron. coutts@uts.ed.au. 